\title{
INFINITESIMAL CONTACT TRANSFORMATIONS AND THEIR APPLICATIONS TO ABSTRACT GROWTH PATTERNS*
}

\author{
BY \\ IGOR FROLOW \\ Oberlin College
}

\begin{abstract}
Several abstract mathematical models of two-dimensional objects or "organisms" are introduced. The objects are abstractions, created by the combination of pattern-theoretic generators. They are deformed by what are shown to be infinitesimal contact transformations. Analytical methods are utilized to determine some of the resulting forms and a computer is programmed to display graphically the developing patterns.
\end{abstract}

1. Introduction. Problems concerned with the development of organisms have long been of interest to man. What are the underlying forces that cause growth and differentiation to occur? What roles do the genes and cytoplasm play? How do initially spherical cells develop into complex, yet similar species with such a high degree of regularity?

During the past century, many mathematical models have been introduced that have been fruitful in the understanding of morphogenesis. Among the many works that should be cited for their brilliance and originality are those of Huxley [6], Thompson [13], Turing [14], Rashevsky [9], Steinberg [11], Lindenmayer [7, 8] and Wolpert [15]. More recently, René Thom has stirred up much excitement and controversy by using catastrophe theory to qualitatively describe the process of morphogenesis (see [12]).

The intent of this paper is to show the possible relevance of infinitesimal transformations in describing the development of an organism. With this in mind, several two-dimensional objects are created and forced to undergo deformations through infinitesimal contact transformations. The objects are abstractions, not true biological organisms. In [4], they are seen as combinations of generators, the basic building blocks of pattern synthesis (see [5]). Much as in D'Arcy Thompson's On growth and form, the emphasis is on the forms of the objects and their development with time. By way of analytical techniques, the "capabilities"' of some of the "organisms" are determined and the theory is supported by computer drawings. For a more extensive study of abstract "organisms" and other types of deformation mechanisms see [4].

2. A brief introduction to contact transformations. The non-homogeneous version of a contact transformation shall be considered, the theory of which was first developed by Sophus Lie at the end of the nineteenth century. A more complete analysis of the nonhomogeneous version as well as of the homogeneous case, may be found in [3].

\footnotetext{
* Received March 14, 1979. This research was supported by the National Science Foundation under grants DCR 74-17921 and MCS 76-80762.

' A word coined by Rosen [10] to refer to the potential patterns that may be generated.
} 
Definition 1. A contact transformation (in two dimensions) is a mapping $\bar{x}=X(x, y, p)$, $\bar{y}=Y(x, y, p), \bar{p}=P(x, y, p)$ from the $x y$ plane to the $\bar{x} \bar{y}$ plane, where $X, Y$ and $P$ are twice continuously differentiable functions with respect to their arguments, such that the Pfaff differential equation

$$
d y-p d x=0
$$

is invariant under the transformation.

If, in particular, $(x, y)$ is a point on a smooth curve $y=y(x)$ and $p$ is the tangent at the point (the point and its tangent forming a so-called line-element), then under the contact transformation the line-element is mapped into a line-element in the $\bar{x} \bar{y}$ plane.

As a consequence of the invariance of $(2.1)$, there exists a function $\gamma(x, y, p)$ such that

$$
d \bar{y}-\bar{p} d \bar{x}=\gamma(x, y, p)(d y-p d x) .
$$

The proof may be seen in [3].

Clearly, if $\gamma(x, y, p)=0$, the contact transformation maps the independent variables into a line-element, regardless of whether the variables form a line-element or not. On the other hand, if $\gamma(x, y, p) \neq 0$, then a mapping into a line-element implies that the independent variables must themselves form a line-element.

It is now possible to state a theorem for determining whether a given mapping is or is not a contact transformation (see [2], p. 114).

THEOREM 1. The mapping $\bar{x}=X(x, y, p), \bar{y}=Y(x, y . p)$, and $\bar{p}=P(x, y, p)$ defines a contact transformation iff

$$
\begin{aligned}
& {[Y, X]=\frac{\partial Y}{\partial p}\left(\frac{\partial X}{\partial x}+p \frac{\partial X}{\partial y}\right)-\frac{\partial X}{\partial p}\left(\frac{\partial Y}{\partial x}+p \frac{\partial Y}{\partial y}\right)=0} \\
& {[P, Y]=\frac{\partial P}{\partial p}\left(\frac{\partial Y}{\partial x}+p \frac{\partial Y}{\partial y}\right)-\frac{\partial Y}{\partial p}\left(\frac{\partial P}{\partial x}+p \frac{\partial P}{\partial y}\right)=\gamma P} \\
& {[P, X]=\frac{\partial P}{\partial p}\left(\frac{\partial X}{\partial x}+p \frac{\partial X}{\partial y}\right)-\frac{\partial X}{\partial p}\left(\frac{\partial P}{\partial x}+p \frac{\partial P}{\partial y}\right)=\gamma}
\end{aligned}
$$

3. A growth model based on infinitesimal tangent rotations. Let us assume that we are given a simply connected, closed, convex curve with a counterclockwise orientation. We shall transform it according to the following principle: if the curve is a polygon with a finite number of sides, each side is rotated $\delta$ degrees about its extreme point $(\delta$ is small in magnitude and unrestricted in sign). The intersection points of the resulting set of rotated sides are determined and connected to form a new curve (see Fig. 1, the arrow in this and subsequent figures is used to identify the configuration resulting from the application of a specific transformation).

If the initial curve is convex and smooth, then the new curve is obtained by finding the envelope (if it exists) of the family of tangent lines rotated $\delta$ degrees about their points of tangency. It should be noted that the first case is a discrete version of the second.

Given a smooth, closed convex curve in the plane, we can parametrize it by an angle $\alpha$ $(0<\alpha<2 \pi)$, the positive angle of rotation between one of the axes (say $x)$ and the tangent at a point (see Fig. 2). We may assume a counterclockwise orientation of the curve.

If the tangent line at a point $(x(\alpha), y(\alpha))$ is rotated by an angle $\delta$, the equation of the line becomes

$$
\bar{y}-y(\alpha)=[\tan (\alpha+\delta)](\bar{x}-x(\alpha))
$$




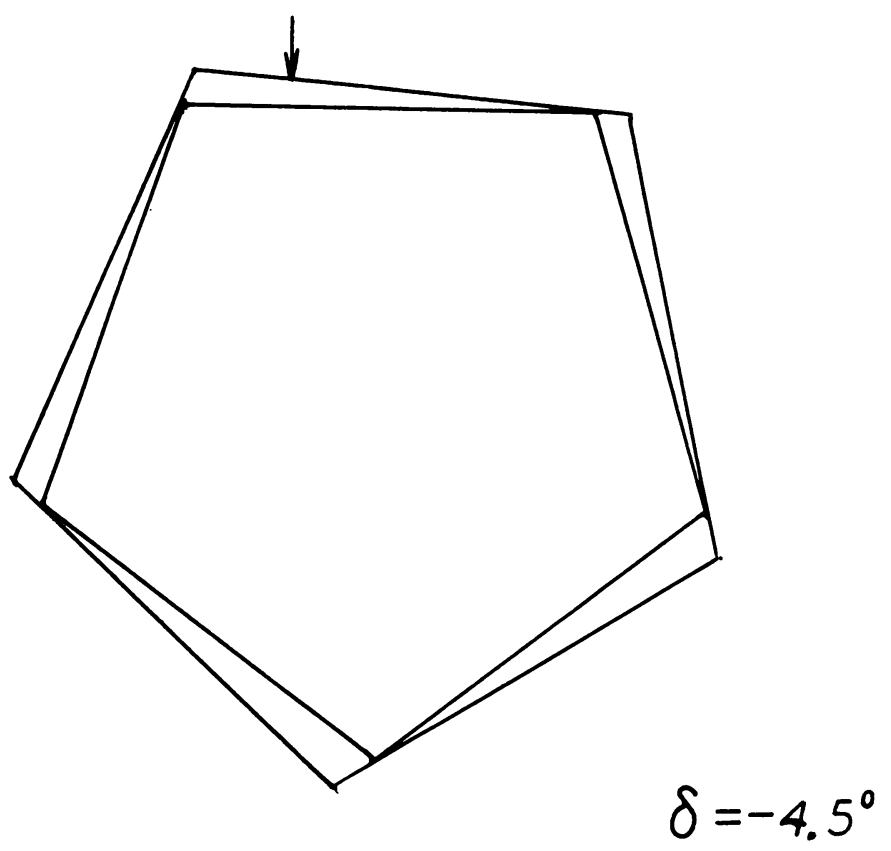

FIG. 1.

As a function of the angle $\alpha$, (3.1) represents a one-parameter family of lines. To compute its envelope, we first differentiate (3.1) with respect to $\alpha$ and obtain

$$
-y^{\prime}(\alpha)=\left[\sec ^{2}(\alpha+\delta)\right][\bar{x}-x(\alpha)]-x^{\prime}(\alpha) \tan (\alpha+\delta) .
$$

We then solve for $\bar{x}$ and $\bar{y}$ from (3.1) and (3.2) and get

$$
\begin{aligned}
& \bar{x}=x(\alpha)+x^{\prime}(\alpha) \frac{\sin \delta \cos (\alpha+\delta)}{\cos \alpha}, \\
& \bar{y}=y(\alpha)+y^{\prime}(\alpha) \frac{\sin \delta \sin (\alpha+\delta)}{\sin \alpha} .
\end{aligned}
$$

For the special case when the smooth curve is a circle, the following interesting result is obtained:

THEOREM 2. Given a circle of radius $R$, the envelope of the set of tangent lines rotated $\delta$ degrees about their points of tangency is again a circle with radius $R \cos \delta$.

Proof. For a circle of radius $R$ centered at the origin, $x(0)=0$ and $y(0)=-R$. The coordinates of all other points on the circle may be determined by applying the orthogonal transformation

$$
\left[\begin{array}{cc}
\cos \alpha & -\sin \alpha \\
\sin \alpha & \cos \alpha
\end{array}\right]
$$

to the column vector

$$
\left[\begin{array}{c}
0 \\
-R
\end{array}\right] \text {, }
$$




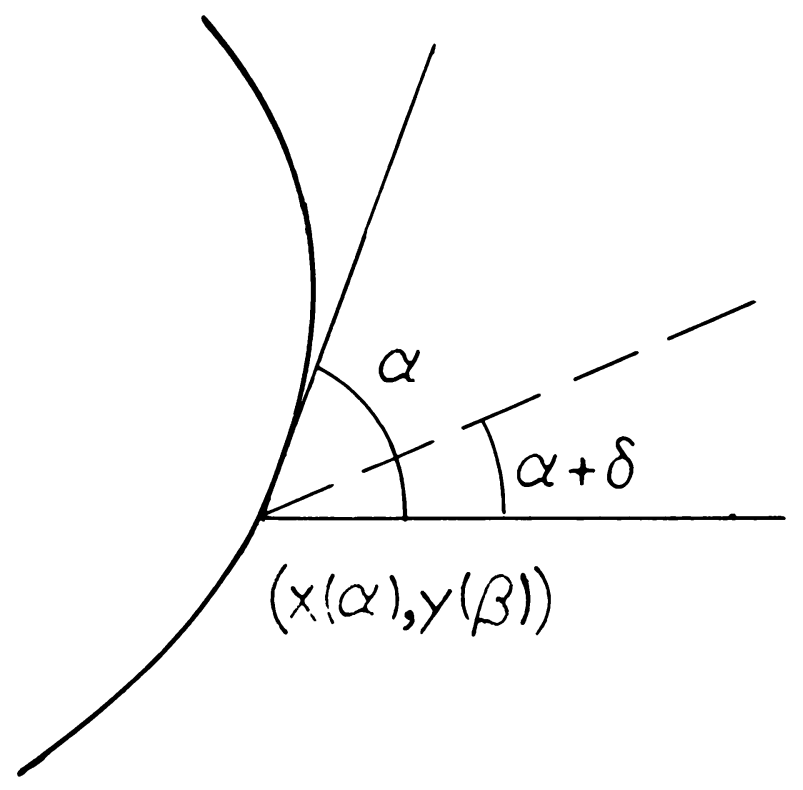

FIG. 2.

where $0<\alpha<2 \pi$. That is,

$$
\left[\begin{array}{l}
x(\alpha) \\
y(\alpha)
\end{array}\right]=\left[\begin{array}{cc}
\cos \alpha & -\sin \alpha \\
\sin \alpha & \cos \alpha
\end{array}\right]\left[\begin{array}{c}
0 \\
-R
\end{array}\right]=\left[\begin{array}{c}
R \sin \alpha \\
-R \cos \alpha
\end{array}\right] .
$$

Differentiating, we get

$$
\left[\begin{array}{ll}
x^{\prime} & (\alpha) \\
y^{\prime} & (\alpha)
\end{array}\right]=\left[\begin{array}{l}
R \cos \alpha \\
R \sin \alpha
\end{array}\right] .
$$

Substituting these results into (3.3) and (3.4), we obtain the following for the coordinates of the envelope:

$$
\begin{gathered}
\bar{x}=R \sin \alpha+R \sin \delta \cos (\alpha+\delta), \\
\bar{y}=-R \cos \alpha+R \sin \delta \sin (\alpha+\delta) .
\end{gathered}
$$

But these are the coordinates of a circle of radius $R \cos \delta$ as can be verified by showing that $\bar{x}^{2}+\bar{y}^{2}=R^{2} \cos ^{2} \delta$.

We thus expect the new circle to lie within the original one, regardless of whether $\delta$ is positive or negative, as is verified by the computer simulations shown in Figs. 3(a) and 3(b). The abstract organism may be said to be experiencing "degrowth" or negative growth.

Given this result, it is reasonable to expect that for the more general case of any closed convex curve, under the transformation, a point $C$ is mapped into a point $C^{\prime}$ on the circle of radius $\rho \cos \delta$, centered at the center of curvature of $C$. The value $\rho$ is the radius of curvature at $C$.

The transformation that we have defined maps a point and its tangent line (i.e. a line- 


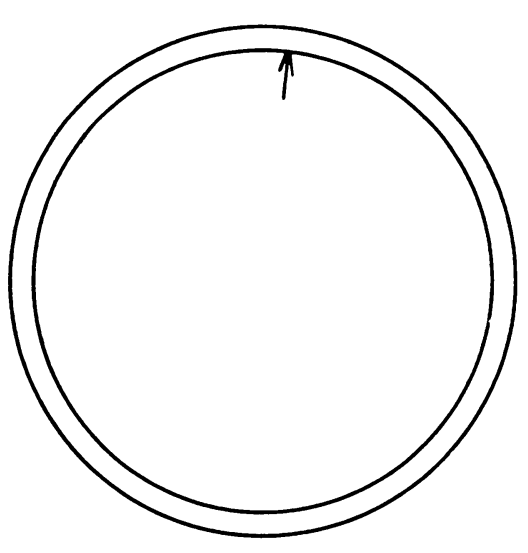

$$
\delta=20^{\circ}
$$

(a)

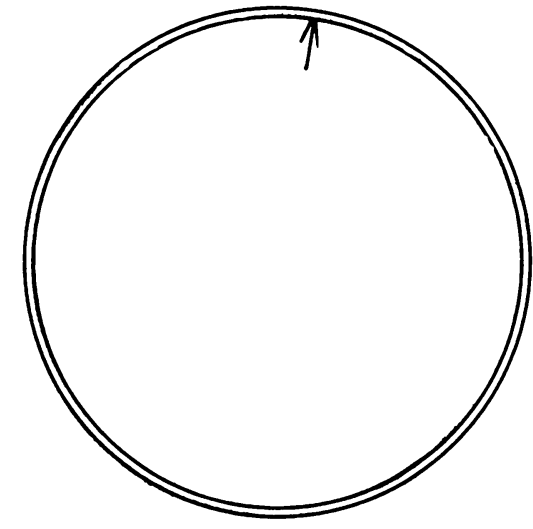

$\delta=-20^{\circ}$

(b)

FIG. 3.

element) into a point on a line. We now show that for the special case when the initial curve is a circle, the transformation is a contact transformation. Using the results of (3.5), (3.6) and (3.7), we get

$$
\begin{aligned}
\bar{x} & =R \sin \alpha+R \sin \delta \cos \alpha \cos \delta-R \sin ^{2} \delta \sin \alpha \\
& =x-y \sin \delta \cos \delta-x \sin ^{2} \delta=x \cos ^{2} \delta-y \sin \delta \cos \delta, \\
\bar{y} & =-R \cos \alpha+R \sin \delta \sin \alpha \cos \delta+R \sin ^{2} \delta \cos \alpha \\
& =y+x \sin \delta \cos \delta-y \sin ^{2} \delta=y \cos ^{2} \delta+x \sin \delta \cos \delta .
\end{aligned}
$$

With

$$
\bar{p}=\tan (\alpha+\delta)=\frac{\tan \alpha+\tan \delta}{1-\tan \alpha \tan \delta}=\frac{p+\tan \delta}{1-p \tan \delta}=\frac{p \cos \delta+\sin \delta}{\cos \delta-p \sin \delta},
$$

we obtain the following relation:

$$
\begin{aligned}
d \bar{y}-\bar{p} d \bar{x}= & \cos ^{2} \delta d y+\sin \delta \cos \delta d x-\left[\frac{p \cos \delta+\sin \delta}{\cos \delta-p \sin \delta}\right]\left[\cos ^{2} \delta d x-\sin \delta \cos \delta d y\right] \\
= & \frac{1}{\cos \delta-p \sin \delta}\left\{\cos ^{3} \delta d y-p \sin \delta \cos ^{2} \delta d y+\sin \delta \cos ^{2} \delta d x\right. \\
& -p \sin ^{2} \delta \cos \delta d x-p \cos ^{3} \delta d x-\sin \delta \cos ^{2} \delta d x \\
& \left.+p \sin \delta \cos ^{2} \delta d y+\sin ^{2} \delta \cos \delta d y\right\} \\
= & \frac{1}{\cos \delta-p \sin \delta}\left\{\left(\cos ^{3} \delta+\sin ^{2} \delta \cos \delta\right)(d y-p d x)\right\}=0
\end{aligned}
$$

whenever $d y-p d x=0$. 
We thus conclude that the transformation

$$
\bar{x}=x \cos ^{2} \delta-y \sin \delta \cos \delta, \quad \bar{y}=y \cos ^{2} \delta+x \sin \delta \cos \delta, \quad \bar{p}=\frac{p \cos \delta+\sin \delta}{\cos \delta-p \sin \delta}
$$

mapping circles into circles is a contact transformation.

4. Characterizing the subclass of contact transformations in a specific family of infinitesimal transformations. Consider the family of infinitesimal transformations (à la Lie)

$$
\left[\begin{array}{c}
\bar{x} \\
\bar{y} \\
\bar{p}
\end{array}\right]=\left[\begin{array}{l}
x \\
y \\
p
\end{array}\right]+\delta\left[M\left[\begin{array}{l}
x \\
y \\
p
\end{array}\right]+v\right]
$$

taking the $x y$ plane into the $\bar{x} \bar{y}$ plane. $\delta$ is some infinitesimal parameter, $M$ is any threeby-three matrix and $v$ is any three-dimensional column vector. The variables $p$ and $\bar{p}$ are directions associated with the points $(x, y)$ and $(\bar{x}, \bar{y})$, respectively. We would like to determine the form that $M$ and $v$ must take in order that (4.1) be a contact transformation.

Let the $(i, j)$ th component of $M$ be $m_{i j}, i, j=1,2,3$ and the $j$ th component of $v$ be $v_{j}$, $j=1,2,3$. Then (4.1) can be written as the system of equations

$$
\begin{aligned}
& \bar{x}=x+\delta\left(m_{11} x+m_{12} y+m_{13} p+v_{1}\right), \\
& \bar{y}=y+\delta\left(m_{21} x+m_{22} y+m_{23} p+v_{2}\right), \\
& \bar{p}=p+\delta\left(m_{31} x+m_{32} y+m_{33} p+v_{3}\right) .
\end{aligned}
$$

If these functions define a contact transformation then, by Theorem 1, Eqs. (2.3), (2.4) and (2.5) must be satisfied, i.e.

$$
\begin{gathered}
{[Y, X]=\delta m_{23}\left(1+\delta m_{11}+p \delta m_{12}\right)-\delta m_{13}\left[\delta m_{21}+p\left(1+\delta m_{22}\right)\right]=0} \\
{[P, Y]=\left(1+\delta m_{33}\right)\left[\delta m_{21}+p\left(1+\delta m_{22}\right)\right]-\delta m_{23}\left(\delta m_{31}+p \delta m_{32}\right)} \\
=\gamma\left[p+\delta\left(m_{31} x+m_{32} y+m_{33} p+v_{3}\right)\right], \\
{[P, X]=\left(1+\delta m_{33}\right)\left(1+\delta m_{11}+p \delta m_{12}\right)-\delta m_{13}\left(\delta m_{31}+p \delta m_{32}\right)=\gamma}
\end{gathered}
$$

Viewing (4.2), (4.3) and (4.4) as polynomial equations in alternately $p, x$, and $y$, it can be shown that $M$ must take on one of two different forms:

$$
\left[\begin{array}{ccc}
0 & 0 & 0 \\
v_{3} & m_{22} & 0 \\
0 & 0 & m_{22}
\end{array}\right] \text { or }\left[\begin{array}{ccc}
m_{22} & 0 & 0 \\
0 & m_{22} & 0 \\
0 & 0 & 0
\end{array}\right]
$$

The converse is also true. That is, if $M$ takes on one of the above forms, (4.1) is a contact transformation and (4.2), (4.3) and (4.4) are satisfied. To see this, let

$$
M=\left[\begin{array}{ccc}
0 & 0 & 0 \\
v_{3} & m_{22} & 0 \\
0 & 0 & m_{22}
\end{array}\right]
$$


Then transformation (4.1), written as a system of equations, becomes

$$
\bar{x}=x+\delta v_{1}, \quad \bar{y}=y+\delta v_{3} x+\delta m_{22} y+\delta v_{2}, \quad \bar{p}=p+\delta m_{22} p+\delta v_{3} .
$$

Solving for $d \bar{y}-\bar{p} d \bar{x}$, we get

$$
\begin{aligned}
d \bar{y}-\bar{p} d \bar{x} & =d y+\delta v_{3} d x+\delta m_{22} d y-\left(p+\delta m_{22} p+\delta v_{3}\right) d x \\
& =\left(1+\delta m_{22}\right)(d y-p d x)=0
\end{aligned}
$$

whenever $d y-p d x=0$. Similarly, if

$$
M=\left[\begin{array}{ccc}
m_{22} & 0 & 0 \\
0 & m_{22} & 0 \\
0 & 0 & 0
\end{array}\right]
$$

then

$$
\begin{aligned}
\bar{x}=x+\delta m_{22} x+\delta v_{1}, & \bar{y}=y+\delta m_{22} y+\delta v_{2}, \quad \bar{p}=p\left(v_{3}=0\right), \\
d \bar{y}-\bar{p} d \bar{x}= & \left(1+\delta m_{22}\right) d y-p\left(1+\delta m_{22}\right) d x \\
= & \left(1+\delta m_{22}\right)(d y-p d x)=0
\end{aligned}
$$

when $d y-p d x=0$.

All of the above results are now summarized in the following theorem. THEOREM 3. The infinitesimal transformation

$$
\left[\begin{array}{c}
\bar{x} \\
\bar{y} \\
\bar{p}
\end{array}\right]=\left[\begin{array}{c}
x \\
y \\
p
\end{array}\right]+\delta\left[M\left[\begin{array}{l}
x \\
y \\
p
\end{array}\right]+v\right] \text { for } 0<|\delta| \ll 1
$$

is a contact transformation if and only if $M$ takes on one of the two different forms

$$
\left[\begin{array}{ccc}
0 & 0 & 0 \\
v_{3} & m & 0 \\
0 & 0 & m
\end{array}\right] \text { or }\left[\begin{array}{ccc}
m & 0 & 0 \\
0 & m & 0 \\
0 & 0 & 0
\end{array}\right] \text {, }
$$

where $v_{3}$ is the third component of $v$ and $m$ is an arbitrary constant.

5. Line-to-line mappings and growth. Suppose that we are given a simply connected, smooth closed curve. At each point $\left(x_{0}, y_{0}\right)$ on the curve, we take the tangent line and rotate it about the point in accordance with an infinitesimal change $d p$ in its slope and the resulting change of $d l$ in its $y$-intercept. Thus, associated with each point is a pair of lines

$$
\begin{gathered}
y=p x+l, \\
y=(p+d p) x+(l+d l) .
\end{gathered}
$$

Suppose that there exists a line-to-line mapping

$$
\bar{p}=\bar{p}(p, l), \quad \bar{l}=\bar{l}(p, l)
$$

such that the functions $\bar{p}$ and $\bar{l}$ are continuously differentiable with respect to their arguments and such that each pair of lines defined by (5.1) and (5.2) are mapped into another 
pair of intersecting lines. By connecting the intersection points $\left(\bar{x}_{0}, \bar{y}_{0}\right)$, a new curve may be obtained (see Fig. 4).

The fact that a point and a direction are mapped into a new point and direction indicates that the transformation is a contact transformation, a result which is stated formally as the following theorem.

THEOREM 4. The line to line mapping

$$
\bar{p}=\bar{p}(p, l)=\bar{p}\left(p, y_{0}-p x_{0}\right), \quad \bar{l}=\bar{l}(p, l)=\bar{l}\left(p, y_{0}-p x_{0}\right)
$$

defines a contact transformation, the transformation taking the coordinates $\left(x_{0}, y_{0}, p\right)$ associated with a pair of intersecting lines of the form (5.1) and (5.2) into $\left(\bar{x}_{0}, \bar{y}_{0}, \bar{p}\right)$, the coordinates associated with the images of (5.1) and (5.2) under (5.4).

Proof. The images of (5.1) and (5.2) under (5.4) are the lines

$$
\begin{gathered}
\bar{y}=\bar{p}(p, l) \bar{x}+\bar{l}(p, l), \\
\bar{y}=\bar{p}(p+d p, l+d l) \bar{x}+\bar{l}(p+d p, l+d l) \\
=\left(\bar{p}+\frac{\partial \bar{p}}{\partial p} d p+\frac{\partial \bar{p}}{\partial l} d l\right) \bar{x}+\left(\bar{l}+\frac{\partial \bar{l}}{\partial p} d p+\frac{\partial \bar{l}}{\partial l} d l\right) \\
=(\bar{p}+d \bar{p}) \bar{x}+(\bar{l}+d \bar{l}),
\end{gathered}
$$

where higher-order terms have been disregarded under the assumption that $d p$ and $d l$ are very small.

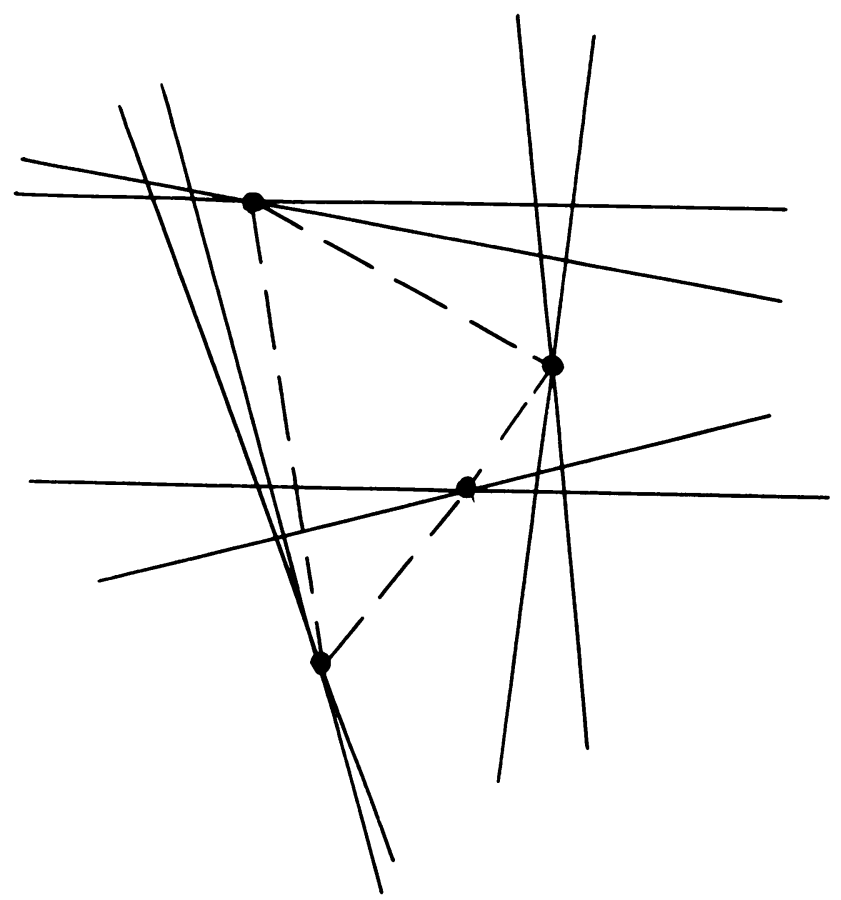

FIG. 4. 
Since the lines are expected to intersect at the point $\left(\bar{x}_{0}, \bar{y}_{0}\right)$, we can solve for $\bar{x}_{0}$ and $\bar{y}_{0}$. Substituting these values into $\bar{x}$ and $\bar{y}$ in (5.5) and (5.6) and then subtracting (5.5) from (5.6), we get

$$
\bar{x}_{0} d \bar{p}+d \bar{l}=0
$$

or, equivalently,

$$
\bar{x}_{0}=-d \bar{l} / d \bar{p}
$$

assuming $\bar{p} \neq$ constant. Furthermore, from (5.5)

$$
\bar{y}_{0}=\bar{l}-(d \bar{l} / d \bar{p}) \bar{p}
$$

We now have

$$
\begin{aligned}
& d \bar{y}_{0}=\frac{\partial \bar{y}_{0}}{\partial p} d p+\frac{\partial \bar{y}_{0}}{\partial l} d l \\
& d \bar{x}_{0}=\frac{\partial \bar{x}_{0}}{\partial p} d p+\frac{\partial \bar{x}_{0}}{\partial l} d l \\
& d l=\frac{\partial l}{\partial x_{0}} d x_{0}+\frac{\partial l}{\partial y_{0}} d y_{0}+\frac{\partial l}{\partial p} d p=-p d x_{0}+d y_{0}-x_{0} d p=-x_{0} d p
\end{aligned}
$$

where, in the last equation, we have assumed that

$$
d y_{0}-p d x_{0}=0 .
$$

Substituting (5.10) into (5.8) and (5.9), we obtain

$$
d \bar{y}_{0}=\frac{\partial \bar{y}_{0}}{\partial p} d p-x_{0} \frac{\partial \bar{y}_{0}}{\partial l} d p, \quad d \bar{x}_{0}=\frac{\partial \bar{x}_{0}}{\partial p} d p-x_{0} \frac{\partial \bar{y}_{0}}{\partial l} d p
$$

so that

$$
d \bar{y}_{0}-\bar{p} d \bar{x}_{0}=\left(\frac{\partial \bar{y}_{0}}{\partial p}-x_{0} \frac{\partial \bar{y}_{0}}{\partial l}-\bar{p} \frac{\partial \bar{x}_{0}}{\partial p}+\bar{p} x_{0} \frac{\partial \bar{x}_{0}}{\partial l}\right) d p
$$

But, according to (5.5),

$$
\bar{y}_{0}=\overline{p x}_{0}+\bar{l},
$$

which, upon differentiation with respect to $p$ and $l$, yields

$$
\frac{\partial \bar{y}_{0}}{\partial p}=\frac{\partial \bar{p}}{\partial p} \bar{x}_{0}+\bar{p} \frac{\partial \bar{x}_{0}}{\partial p}+\frac{\partial \bar{l}}{\partial p}, \quad \frac{\partial \bar{y}_{0}}{\partial l}=\frac{\partial \bar{p}}{\partial l} \bar{x}_{0}+\bar{p} \frac{\partial \bar{x}_{0}}{\partial l}+\frac{\partial \bar{l}}{\partial l}
$$

Thus, given these results, (5.11) becomes

$$
d \bar{y}_{0}-\bar{p} d \bar{x}_{0}=\left[\left(\frac{\partial \bar{l}}{\partial p}-x_{0} \frac{\partial \bar{l}}{\partial l}\right)+\bar{x}_{0}\left(\frac{\partial \bar{p}}{\partial p}-x_{0} \frac{\partial \bar{p}}{\partial l}\right)\right] d p .
$$

Substituting $-d \bar{l} / d \bar{p}$ for $\bar{x}_{0}$ from (5.7), we get the final result

$$
d \bar{y}_{0}-\bar{p} d \bar{x}_{0}=\left[\left(\frac{\partial \bar{l}}{\partial p}-x_{0} \frac{\partial \bar{l}}{\partial l}\right)-\frac{d \bar{l}}{d \bar{p}}\left(\frac{\partial \bar{p}}{\partial p}-x_{0} \frac{\partial \bar{p}}{\partial l}\right)\right] d p=0 .
$$




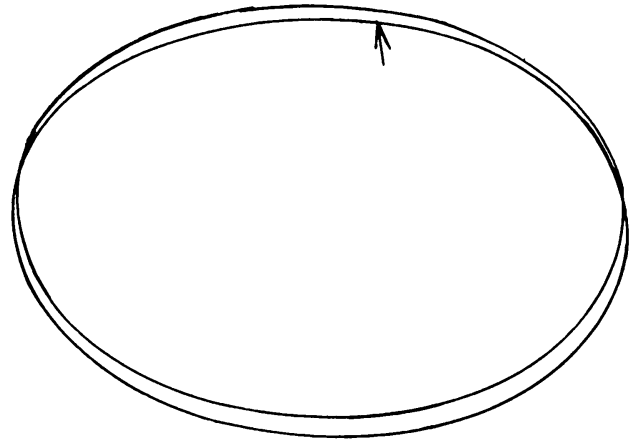

(a) $d p=.01, \epsilon=.01, \Gamma=\left(\begin{array}{ll}1 & 2 \\ 2 & 1\end{array}\right), \gamma=\left(\begin{array}{l}1 \\ 1\end{array}\right)$.

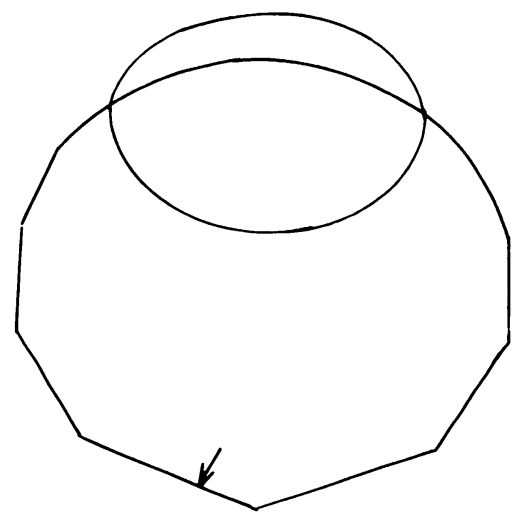

(c) $d p=.01, \epsilon=.1, \Gamma=\left(\begin{array}{c}1 \\ 2 \\ 2\end{array}\right), \gamma=\left(\begin{array}{l}0 \\ 0\end{array}\right)$.

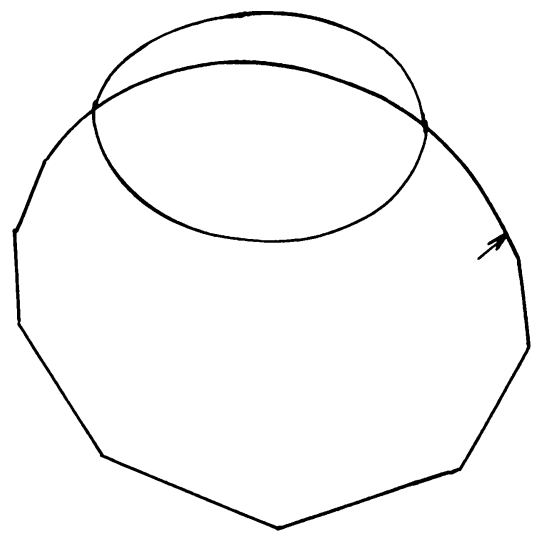

(b) $d p=.01, \epsilon=.1, \Gamma=\left(\begin{array}{ll}1 & 2 \\ 2 & 1\end{array}\right), \gamma=\left(\begin{array}{l}1 \\ 1\end{array}\right)$.

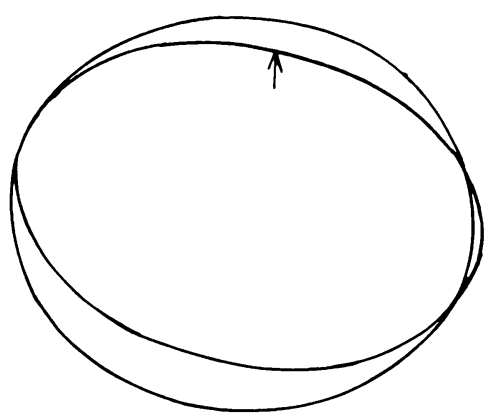

(d) $d p=.01, \epsilon=.2, \Gamma=\left(\begin{array}{l}1 \\ 1 \\ 0\end{array}\right), \gamma=\left(\begin{array}{l}1 \\ 1\end{array}\right)$.

FIG. 5. 
In order to see the types of new curves that could result from a line-to-line map applied to the pairs of intersecting lines associated with the points on an initial curve, we choose the transformation to be of the form

$$
\left[\begin{array}{l}
\bar{p} \\
\bar{l}
\end{array}\right]=\left[\begin{array}{l}
p \\
l
\end{array}\right]+\epsilon\left[\Gamma\left[\begin{array}{l}
p \\
l
\end{array}\right]+\gamma\right]
$$

where $\epsilon$ is some small parameter, $\Gamma$ is a two-by-two matrix and $\gamma$ is a two-dimensional vector. The results for some specific values of $\epsilon, \Gamma$, and $\gamma$ are shown in Figs. 5(a)-5(d).

6. Conclusions. We have observed a few examples of abstract organisms undergoing deformations according to specific mathematical rules. The models appear to be estranged from the complex biochemical processes that are involved during the development of true organisms. However, they do point to the potential of infinitesimal transformations for describing changes in form. Further research is warranted and should concentrate on coordinate-free transformations if the models are to more accurately portray real growth.

\section{REFERENCES}

[1] V. G. Boltianskii, Ogibaiushchaia, Gosudarstvennoe Izdatelstvo Fizico-Mathematicheskoy Literaturi, Moscow, 1961

[2] C. Caratheodory, Calculus of variations and partial differential equations of the first order, Part I (translated by R. B. Dean and J. J. Brandstatter), Holden-Day, San Francisco, 1965

[3] L. P. Eisenhart, Contact transformations, Annals Math. 2 series 30, 211-249 (1928)

[4] I. Frolow, Abstract growth patterns, Ph.D. Thesis, Brown University, 1978

[5] U. Grenander, Pattern synthesis-lectures in pattern theory, Vol. I, Springer-Verlag, New York, 1976

[6] J. S. Huxley, Problems of relative growth, Methuen, London, 1932

[7] A. Lindenmayer, Mathematical models for cellular interactions in development, Part I: filaments with onesided inputs, J. Theor. Biol. 18, 280-299 (1968)

[8] A. Lindenmayer, Mathematical models for cellular interactions in development, Part II: simple and branching filaments with two-sided inputs, J. Theor. Biol. 18, 300-315 (1968)

[9] N. Rashevsky, Mathematical biophysics: physico-mathematical foundations of biology, Vol. I, 3rd ed., Dover, New York, 1960

[10] R. Rosen, Morphogenesis, in Foundations of mathematical biology, Vol. II (R. Rosen, ed.), 1972, 1-77

[11] M. S. Steinberg, Reconstruction of tissues by dissociated cells, in Mathematical models for cell rearrangement (G. D. Mostow, ed.), 1975, 82-99

[12] R. Thom, Structural stability and morphogenesis (translated by D. H. Fowler), W. A. Benjamin, Reading, Massachusetts, 1975

[13] D'Arcy Thompson, On growth and form, 2nd ed., Cambridge University Press, 1942

[14] A. M. Turning, The chemical basis of morphogenesis, Phil. Trans. Roy. Soc. London B237, 37-72 (1952)

[15] L. Wolpert, Positional information and pattern formation, in Toward a theoretical biology, Vol. III (C. H. Waddington, ed.). 1970, 198-230 